\title{
A Pigmented Mycelial Antibiotic in Streptomyces coelicolor: Control by a Chromosomal Gene Cluster
}

\author{
By BRIAN A. M. RUDD† AND DAVID A. HOPWOOD* \\ John Innes Institute, Colney Lane, Norwich NR4 7UH
}

(Received 28 February 1980)

\begin{abstract}
Streptomyces coelicolor was found to produce a third secondary metabolite, in addition to the antibiotics methylenomycin $\mathrm{A}$ and actinorhodin previously described. This is a red pigmented, highly non-polar compound with antibiotic activity against certain Grampositive bacteria. Mutants lacking the red compound fell into five cosynthetic classes. Representatives of each of the five classes were mapped to the chromosome of the producing organism, in a closely linked cluster. Genetic studies provided evidence that this new metabolite is distinct from actinorhodin and indicated that the two pigments do not share parts of the same biosynthetic pathway.
\end{abstract}

\section{INTRODUCTION}

Streptomyces coelicolor A3(2) produces the antibiotics methylenomycin A (Wright \& Hopwood, 1976a; Kirby \& Hopwood, 1977) and actinorhodin (Wright \& Hopwood, 1976b). During a genetic study of actinorhodin biosynthesis (Rudd \& Hopwood, 1979), the organism was found to produce a third secondary metabolite, a red mycelial pigment with a limited antibiotic activity. We report here the isolation of various classes of mutants defective in production of this pigment, mapping of the mutations to a short segment of the chromosomal linkage map, and the demonstration by genetic tests that biosynthesis of this pigment is unrelated to that of actinorhodin.

\section{METHODS}

General. Minimal medium (MM), complete medium (CM) and general cultural and genetic techniques were those described by Hopwood (1967). Nutrient agar (NA) was Oxoid nutrient agar. R2 agar was that of Okanishi et al. (1974). Ultraviolet mutagenesis of spore suspensions was carried out using a $254 \mathrm{~nm}$ germicidal lamp at a dose rate of approximately $4.4 \mathrm{~J} \mathrm{~m}^{-2} \mathrm{~s}^{-1}$. The usual dose was $704 \mathrm{~J} \mathrm{~m}^{-2}$, giving a survival of approximately $1 \%$.

Strains. All Streptomyces coelicolor strains were mutational or recombinational derivatives of strain A3(2) (Hopwood et al., 1973) (Table 1). The locations of markers on the circular linkage map are shown in Fig. 1. Micro-organisms used in testing for sensitivity to the red pigment were those listed by Wright \& Hopwood (1976a).

Cosynthesis tests of red mutants. Plates carrying a series of red mutants were replica-plated to lawns of other red mutants on plates of CM or R2 agar in order to detect production of red pigment in pairwise combinations. Those pairs which gave a positive result were re-examined in tests in which mutants were inoculated on $\mathrm{CM}$ a few millimeters apart in order to characterize the mutants as secretor or converter. In some combinations there was cosynthesis of red pigment in the first test but not in the second in which the two mutants were not in contact with each other.

Tests of antibacterial activity. Plugs $(7 \mathrm{~mm}$ diam.) were cut from $7 \mathrm{~d}$ old confluent $\mathrm{CM}$ plate cultures of

$\uparrow$ Present address: Department of Medicinal Chemistry and Pharmacognosy, School of Pharmacy and Pharmacal Sciences, Purdue University, West Lafayette, Indiana 47907, U.S.A. 
Table 1. Derivatives of Streptomyces coelicolor A3(2)

All strains were from the John Innes Institute culture collection.

\begin{tabular}{|c|c|c|}
\hline Strain no. & Genetic markers & Plasmid status \\
\hline A700 & proAl $\arg A 1$ cys DI8 & SCP1- SCP2+ \\
\hline B18 & his $A 1$ uraAl str $A 1$ act -118 & SCP1- SCP2+ \\
\hline B229† & hisA1 uraA1 strA1 act -118 red-60 & SCP1- SCP2+ \\
\hline B244 & proA1 arg $A 1$ cysD 18 guaA1 act-118 & SCP1- SCP2* \\
\hline B269 & $\arg A 1$ guaA1 act-117 & SCP1- SCP2* \\
\hline B306 & $\arg A 1$ guaAl act -177 & SCP1- SCP2* \\
\hline B307 & $\arg A 1$ guaA1 act -178 & SCP1-SCP2* \\
\hline B309 & $\arg A 1$ guaA1 act-105 & SCP1- SCP2* \\
\hline B314 & hisA1 guaA1 act-118 red-60 & SCP1-SCP2* \\
\hline B317 & $\arg A 1$ cysD 18 guaAl act -118 red- 60 & SCP1-SCP2* \\
\hline B369 & leuB5 mthB2 guaAl act-118 & SCP1- SCP2* \\
\hline B395 & proA1 arg A1 cysD18 strA1 act-118 red-1 & SCP1- SCP2* \\
\hline B396 & pro $A 1$ arg $A 1$ cys $D 18$ str $A 1$ act -118 red-7 & SCP1- SCP2* \\
\hline B397 & proA1 arg $A 1$ cys $D 18$ str $A 1$ act-118 red-13 & SCP1-SCP2* \\
\hline B398 & pro $A 1$ arg $A 1$ cys $D 18$ str $A 1$ act -118 red-17 & SCP1- SCP2* \\
\hline B701† & his 11 uraA1 strA1 act -118 red-1 & SCP1- SCP2 + \\
\hline B $707 \uparrow$ & hisA1 uraAl strAl act-118 red-7 & SCP1- SCP2 + \\
\hline B713† & his $A 1$ uraAl str $A 1$ act -118 red-13 & SCP1- SCP2+ \\
\hline B $717 \dagger$ & his $A 1$ uraAl str $A 1$ act -118 red-17 & SCP1- SCP2+ \\
\hline
\end{tabular}

$\dagger$ Mutant derivatives of strain B18 isolated in the course of this work.

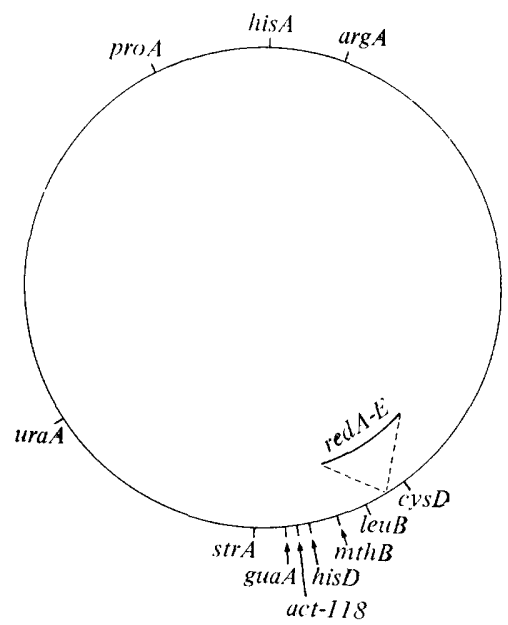

Fig. 1. Linkage map of S. coelicolor A3(2) showing locations of the markers referred to in this paper.

red mutants and other strains, embedded in NA seeded with indicator organisms, and incubated overnight at the optimal growth temperature of the indicator.

Extraction of pigments from mycelium. Strains were grown on $60 \mathrm{~mm}$ diam. circles of dialysis membrane on the surface of CM agar. The mycelium was scraped off and extracted once with 10 to $20 \mathrm{ml}$ methanol. The filtered extract was concentrated to about one-tenth volume by rotary evaporation.

Thin-layer chromatography (t.l.c.). Crude methanol extracts from mycelium were analysed on pre-coated silica gel plates $(5 \times 20 \mathrm{~cm}, 0.25 \mathrm{~mm}$ thick, silica gel 60, no. 5724; Merck, Darmstadt, Germany). Solvent A was chloroform/methanol $(70: 30, \mathrm{v} / \mathrm{v})$; solvent $\mathrm{B}$ was chloroform/methanol $(99: 1, \mathrm{v} / \mathrm{v})$.

\section{RESULTS}

\section{Preliminary evidence for a new pigment}

In a study of mutants blocked in actinorhodin biosynthesis (act mutants), seven phenotypic classes were recognized (Rudd \& Hopwood, 1979). Five of these classes (III to VII) 


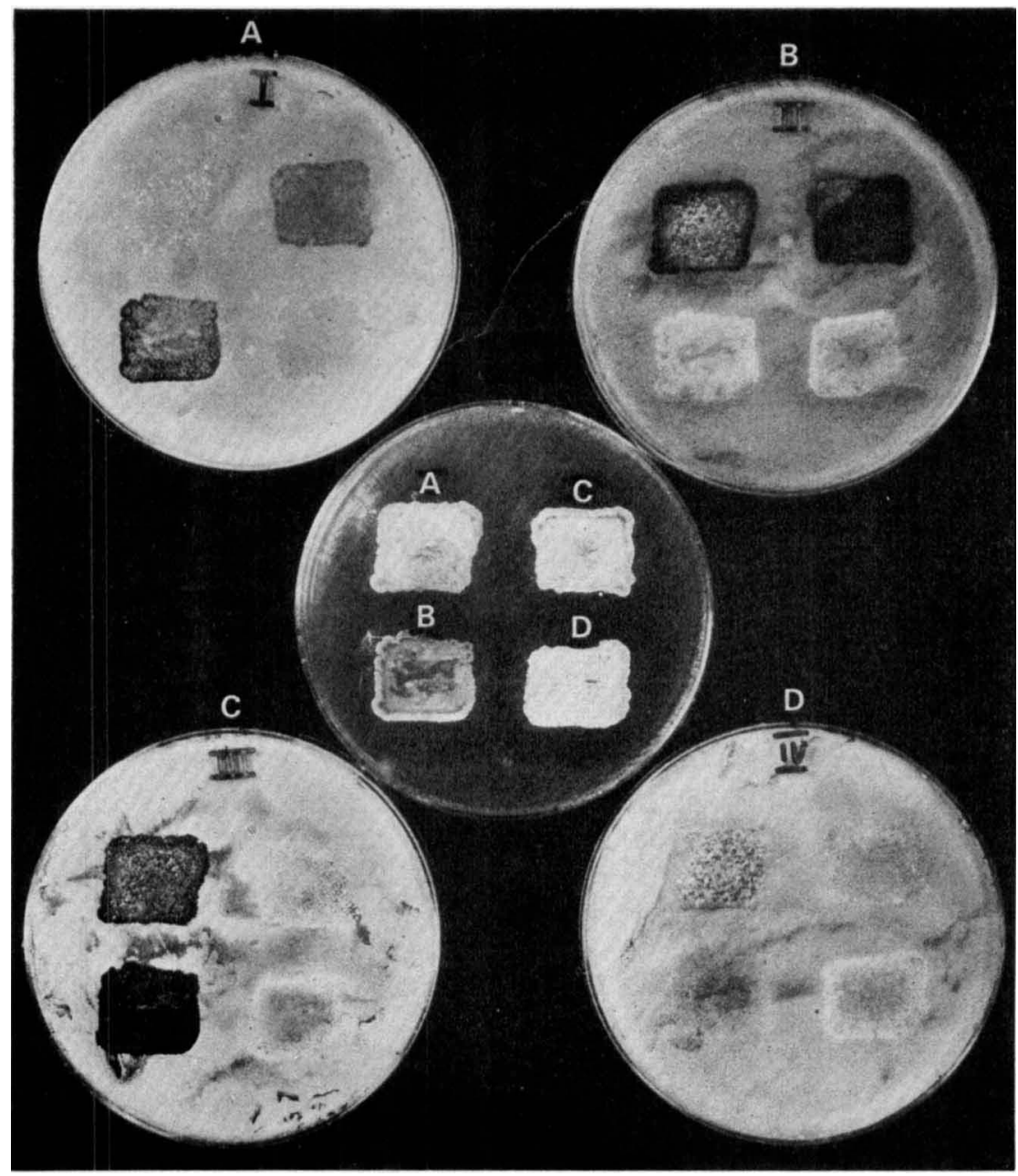

Fig. 2. Examples of cosynthesis between red mutants. The central plate, with four red mutants of different classes, was replicated to lawns of the same four mutants on R2 agar. A, B707 (class A); B, B717 (class B); C, B701 (class C); D, B713 (class D). Cosynthesis is shown by dark patches of pigment synthesis.

produced diffusible pigments, presumably precursors or shunt products of actinorhodin, which is a pigmented antibiotic with acid-base indicator properties. Mutants of all classes except one (class VI) possessed a red pigment in the mycelium when grown on CM or MM. During growth the pigment remained associated with the mycelium rather than diffusing into the medium. On t.l.c. this pigment had an $R_{F}$ of 0.84 in solvent $\mathrm{A}$ and 0.59 in solvent $\mathrm{B}$. In mutants of act classes I and II, which produced no diffusible pigment, this red compound was the major pigment in crude methanol extracts of mycelium.

Members of the one class of act mutants (class VI) in which t.l.c. did not reveal any red mycelial pigment in extracts of cultures grown on CM could be shown to produce red mycelial pigment when grown on NA with added calcium salts (e.g. $4 \mathrm{~mm}$ ); on this medium wild-type $\left(a c t^{+}\right)$strains produced little actinorhodin and act mutants of classes III to VII made little or none of their characteristic diffusible pigment.

Thus, under appropriate conditions, all act mutants could be shown to accumulate a red pigment in their mycelium. 
Table 2. Classes of red mutants and their cosynthetic reactions

\begin{tabular}{|c|c|c|c|c|}
\hline $\begin{array}{l}\text { red mutant } \\
\text { class }\end{array}$ & $\begin{array}{l}\text { No. of mutants } \\
\text { isolated }\end{array}$ & Type strain & $\begin{array}{l}\text { red allele of } \\
\text { type strain }\end{array}$ & Cosynthetic reactions \\
\hline $\mathbf{A}$ & 20 & B707 & red-7 & Converter to classes $\mathrm{B}, \mathrm{C}, \mathrm{E}$ \\
\hline $\mathbf{B}$ & 5 & B717 & red-17 & Secretor to A; cosynthesizes with $\mathrm{C}^{*}$ \\
\hline $\mathrm{C}$ & 5 & B701 & red-1 & Secretor to A; cosynthesizes with $\mathrm{B}^{*}$ \\
\hline $\mathrm{D}$ & 4 & B713 & red-13 & None \\
\hline $\mathrm{E}$ & 3 & B229 & red-60 & Secretor to A \\
\hline
\end{tabular}

Table 3. Numbers and classes of the red mutants isolated from various act parents

$\begin{array}{cccccccr}\text { Parental strain } & \text { act } \text { class } & \text { A } & \text { B } & \text { C } & \text { D } & \text { E } & \text { Total } \\ \text { B18 } & \text { I } & 8 & 3 & 4 & 3 & 2 & 20 \\ \text { B307 } & \text { I } & 2 & 0 & 0 & 0 & 0 & 2 \\ \text { B309 } & \text { II } & 8 & 2 & 0 & 0 & 1 & 11 \\ \text { B306 } & \text { II } & 0 & 0 & 1 & 1 & 0 & 2 \\ \text { B269 } & \text { IV } & 2 & 0 & 0 & 0 & 0 & 2 \\ \text { Total } & & 20 & 5 & 5 & 4 & 3 & 37\end{array}$

\section{Isolation of mutants blocked in red pigment biosynthesis}

Mutants (red) lacking the red mycelial pigment were isolated from five act mutants, belonging to classes I, II and IV, at a frequency of approximately $0 \cdot 2 \%$ after u.v.-irradiation. Only act mutants producing little (class IV) or no diffusible pigment (classes I and II) were suitable as parents since actinorhodin or related compounds obscured red pigment accumulation in the mycelium. The mutants were recognized as yellowish colonies lacking the normal red mycelial colour on CM plates carrying 500 to 1000 colonies.

Some pairs of red mutants cosynthesized red pigment when grown in mixed culture (Fig. 2); on the basis of these cosynthetic reactions the 37 red mutants fell into five classes, A to $\mathrm{E}$ (Table 2). The numbers of mutants of each class isolated from each parental strain are shown in Table 3. The polarity of some, but not all, of the cosynthetic reactions could be recognized: mutants of class $\mathrm{A}$ acted as converters with mutants of classes $\mathrm{B}, \mathrm{C}$ and $\mathrm{E}$, which acted as secretor strains. Intimate mixtures of mutants of classes $B$ and $C$ cosynthesized red pigment, but a polarity in this reaction could not be detected since no pigment was produced when the two strains were separated by a short distance on the agar surface.

The red mutants from the five act parents showed no alteration in act phenotype compared with the parental strains (Rudd \& Hopwood, 1979): the red act strains showed precisely the same cosynthetic reactions with respect to actinorhodin as their red ${ }^{+}$act parental strains; and the red mutants isolated in act class IV strains still produced small quantities of reddishbrown diffusible pigment characteristic of class IV mutants.

\section{Genetic analysis of red mutants}

The type strain of each class of red mutant was selected for genetic mapping. All strains used in these experiments carried act-118 in order to avoid confusion of the red + red classification by the presence of actinorhodin. One or both parents in all the crosses contained the variant SCP2* of the SCP2 sex plasmid, resulting in rather fertile, non-polarized crosses (Bibb et al., 1977; Bibb, 1978) which were very suitable for mapping. Type strains of classes $\mathrm{A}, \mathrm{B}, \mathrm{C}$ and D (strains B707, B717, B701 and B713, respectively, all carrying markers his A1 uraA1 str Al) were crossed with strain B244 (proA1 $\arg A 1$ cysD18 guaAl) and recombinants inheriting his $A^{+}$and $\operatorname{str} A l$ were selected. The results (data not shown) clearly indicated a map location for each of the four red alleles on the chromosome between cys $D$ and guaA. 

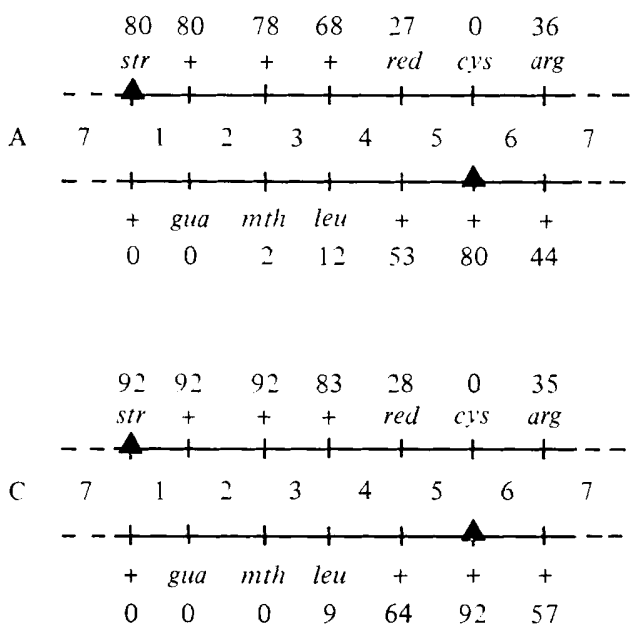
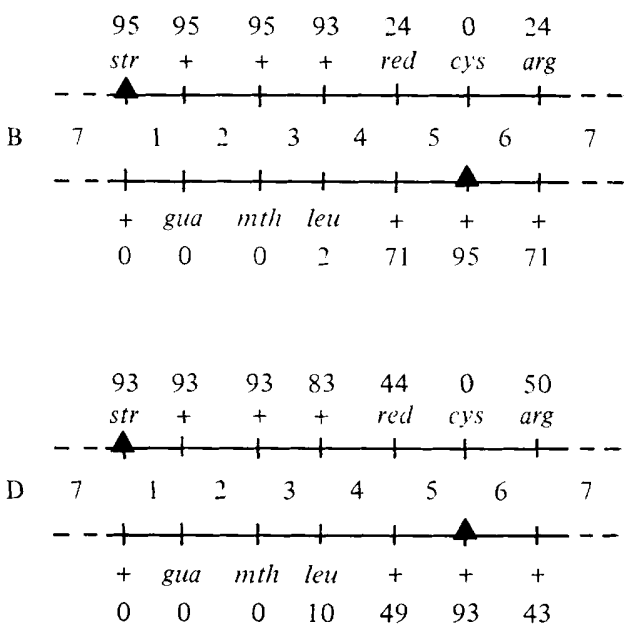

Fig. 3. Allele frequencies in crosses (Table 4) of strain B369 (leuB5 mthB2 guaA1 act-118) with recombinant strains carrying type mutations of red classes $\mathrm{A}$ to $\mathrm{D}$ (with markers proA1 arg $A 1$ cysD18 strA1 act-118). Capital letters indicate the class of the red mutation introduced into the cross in the following parent strains: A, B396; B, B398; C, B395; D, B397. Selection was made for cys $D^{+}$and $\operatorname{str} A 1$ (indicated by triangles). The pro $A 1$ and act-118 markers have been ignored.

Table 4. Results of crosses of strain B369 with recombinant strains carrying the type mutations of red classes $A$ to $D$ (see Fig. 3)

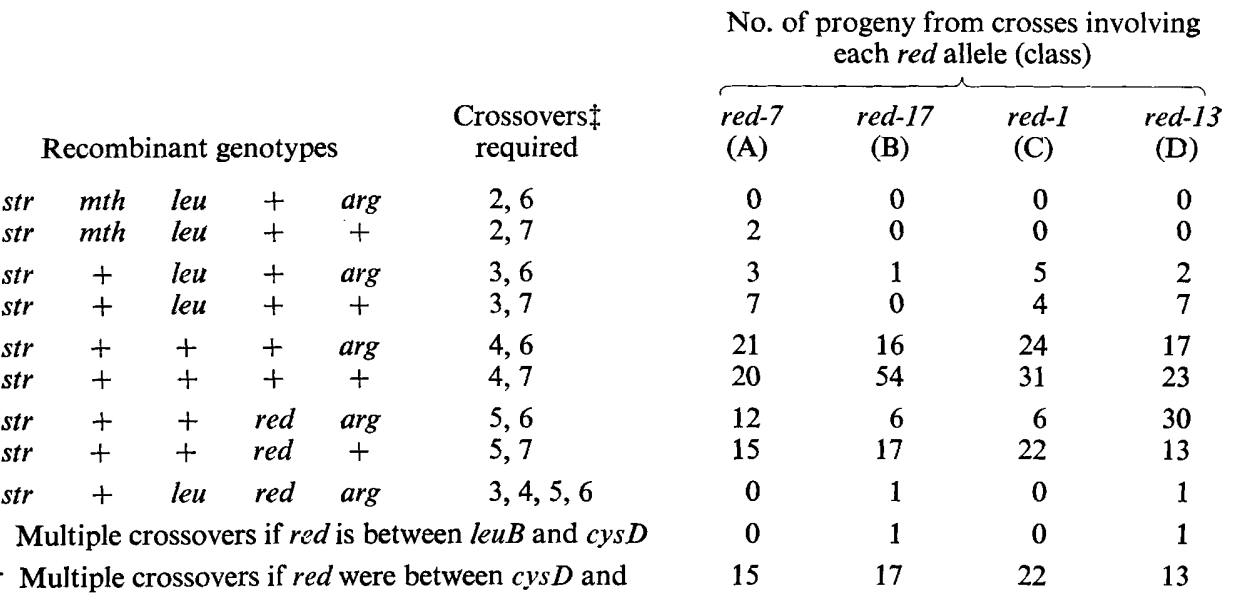

\$ See map intervals in Fig. 3, assuming red is between leuB and cys $D$.

For more precise mapping, four recombinant strains bearing the markers proA1 argAI cys $D 18$ strA1 and each carrying a different red allele (red-7, red-17, red-1 or red-13: strains B396, B398, B395 and B397, respectively) were crossed with strain B369 (leuB5 mthB2 guaAl). The allele ratio for $\mathrm{red}^{+} / \mathrm{red}$ (Fig. 3) was compatible with two alternative locations of the red mutations, between $c y s D$ and $l e u B$ or between $c y s D$ and $\arg A$. The latter location was excluded by the data since it would have required relatively high frequencies of multiple crossover recombinants (Table 4).

Several crosses were made to map the type mutation of class E, red-60. As in the cases of the other red alleles, early crosses gave a location for red-60 between cys D and guaA. 


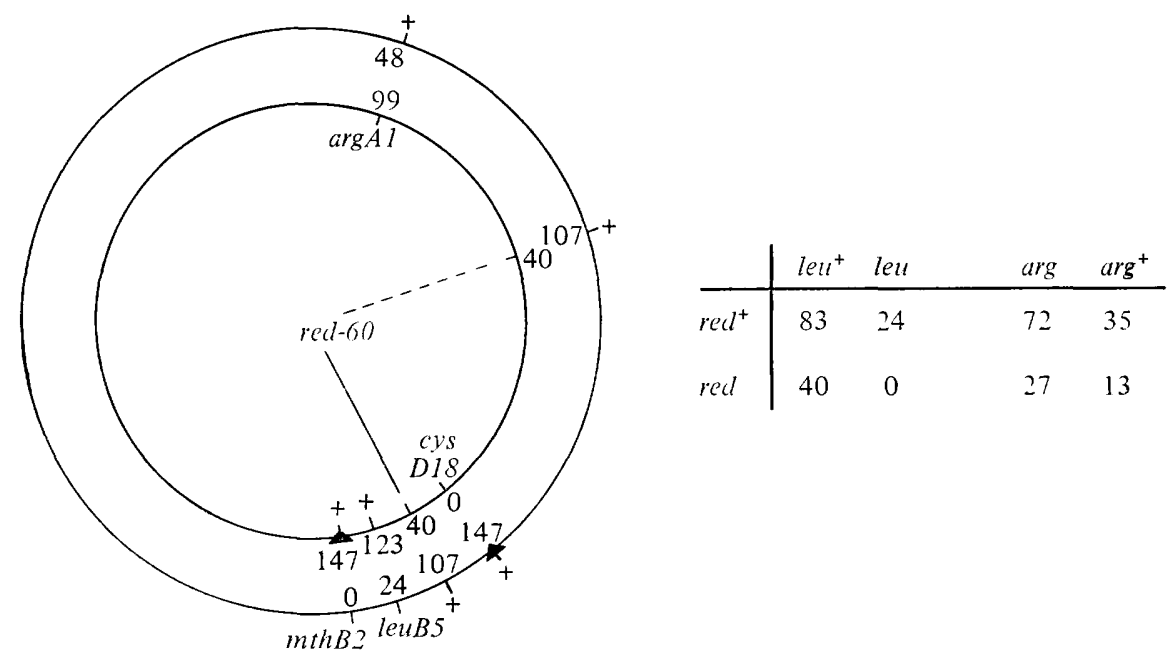

Fig. 4. Analysis of a cross between strains B317 [ $\arg A 1$ guaAl cysD 18 act-118 red-60 (class E)] and $\mathrm{B} 369$ [leuB5 $m t h B 2$ guaAl act-118] with selection for $c y s D^{+}$and $m t h B^{+}$(indicated by triangles). The two alternative locations for red-60, compatible with the observed allele frequencies, are discriminated by the tabulations of the segregations of $\mathrm{red}^{+} / \mathrm{red}$ with respect to $\mathrm{leu}^{+} / \mathrm{leu}$ and $\mathrm{arg}^{+} / \mathrm{arg}$; the data indicate a position for red 60 between cys $D$ and leuB since segregation at the red locus is strongly correlated with that at the $l e u B$ locus but independent of segregation at the $\arg A$ locus.

A further cross, of a recombinant (B317) bearing red-60 and markers $\arg A 1$ cys D18 guaAl, with B369, gave data which located red-60 between $c y s D$ and $l e u B$ (Fig. 4).

Thus the type mutations of all five red classes were unambiguously located in the relatively short chromosomal region between $c y s D$ and $l e u B$, somewhat removed from the actinorhodin cluster which is between his D and guaA (Rudd \& Hopwood, 1979).

\section{Phenotypes of red-60 in combination with other act mutations and act $t^{+}$}

To examine further the apparent independence of the red pigment and actinorhodin, double mutant strains carrying red-60 with act mutations of classes II, III, IV, V and VI were prepared. This was achieved by crossing act strains carrying cys $D$ (which is closely linked to the red alleles) with the red-60 strain B314 bearing the guaA allele, which is closely linked to the act cluster. Selection of $c y s D^{+} g u a A^{+}$yielded recombinants carrying red-60 together with each act mutation at high frequency. Similarly, a cross between the $a c t^{+}$strain A700 (proAl $\arg A 1$ cys D18) and B314 gave an act ${ }^{+}$red-60 recombinant on selection for cys $D^{+} g u a A^{+}$. The absence of red pigment production in these strains was demonstrated by t.l.c., and also by growing them on NA with $4 \mathrm{mM}-\mathrm{Ca}\left(\mathrm{NO}_{3}\right)_{2}$, in order to depress the production of actinorhodin and pigments related to it but to enhance any production of red pigment that might have occurred.

The phenotypes, in respect of the actinorhodin pathway, of strains of act classes II, III, IV, V and VI carrying red-60 were unchanged when compared with the parent red ${ }^{+}$act strains: their diffusible pigments (where relevant) and cosynthetic reactions were those expected (Rudd \& Hopwood, 1979). Similarly, an act ${ }^{+}$red-60 strain produced normal levels of actinorhodin while having no red mycelial pigment.

\section{Nature and antibiotic properties of the red pigment}

The structure of the red pigment has not been elucidated. It appears to be highly non-polar, being almost insoluble in water but soluble in most organic solvents. In dilute alkaline solutions the pigment gives a yellow colour, turning to red in acidic solutions. In tests for antimicrobial activity of $\mathrm{red}^{+}$and $\mathrm{red}$ strains, $\mathrm{red}^{+}$strains gave minute (1 mm or less) zones 
of inhibition of certain Bacillus spp., including B. mycoides and B. cereus. No red mutant showed any inhibition of these strains. No activity was noted against any of the other microorganisms tested. The small zone size noted in the antibiotic assay used may well refiect the poor diffusibility of the red pigment in agar.

Recent work on the chemistry of the red mycelial pigment (S. W. Lee, C.-J. Chang, B. A. M. Rudd \& H. G. Floss, unpublished data) indicates that the pigment is similar to prodigiosin-like pigments produced by other Streptomyces strains, including an actinorhodinproducing strain of $S$. coelicolor (see, for example, Gerber \& Le Chevalier, 1976). Prodigiosin is a red pigment produced by Serratia marcescens. Prodigiosin and related (prodiginine) compounds produced by Streptomyces spp. and other micro-organisms have been reported to possess activity against Gram-positive bacteria (Gerber, 1975). Structural elucidation of the red pigment is in progress.

\section{DISCUSSION}

On the evidence of its genetics, the red mycelial pigment appears to be a new secondary metabolite of $S$. coelicolor A3(2). Genetically it is distinct from actinorhodin on the basis of several criteria: mutations abolishing red pigment production did not affect the act phenotype of parent strains; a red allele (red-60) could be crossed into act mutants of most classes and into $\mathrm{act}^{+}$strains, with no effect on the act phenotype; all red mutants so far investigated mapped to a short segment of the chromosome between $c y s D$ and leuB; and no class of mutant was discovered in the search for act mutations (Rudd \& Hopwood, 1979) that was both fully Act ${ }^{-}$and $\mathrm{Red}^{-}$, apart from strains which can reasonably be assumed to be pleiotropically defective in secondary metabolite production, such as bld mutants which have no aerial mycelium (Merrick, 1976). Thus, it is unlikely that the red pigment is a precursor of actinorhodin, or that actinorhodin and the red pigment are end-products of a branched pathway. If there is a common precursor it must be a very early one.

Of the five classes of mutants blocked in red pigment production isolated in this study, one (class D) was not implicated in cosynthesis with other mutants. A similar situation exists with the classes of act mutants of S. coelicolor, in which mutants of one class (class II) showed no cosynthetic reactions with mutants of other classes (Rudd \& Hopwood, 1979). This class may represent mutations in a regulatory gene. The order in which the genes represented by red mutants of classes $\mathrm{B}, \mathrm{C}$ and $\mathrm{E}$ acted in the biosynthesis of the red pigment could not be elucidated, since although mutants of classes $B$ and $C$ cosynthesized red pigment in mixed culture, it was not possible to demonstrate which mutants acted as secretor and which as converter. Mutants of class A acted as converter to mutants of classes B, C and E and were thus revealed to be early-blocked mutants.

The finding that genes controlling the biosynthesis of this new antibiotic in $S$. coelicolor, like those for actinorhodin (Rudd \& Hopwood, 1979), appear to be closely linked in a single chromosomal cluster strengthens the idea that genes for antibiotic synthesis in streptomycetes may generally be organized in clusters, perhaps corresponding to operons. This generalization, if confirmed, has obvious implications in attempts to transfer the genes for complete antibiotic pathways, or parts of them, from one species to another for analytical purposes or with the objective of developing strains capable of making new antibiotics.

B. A. M. Rudd gratefully acknowledges a graduate studentship provided by Pfizer Ltd.

\section{REFERENCES}

BIBв, M. J. (1978). Genetical and physical studies of a Streptomyces coelicolor plasmid. Ph.D. thesis, University of East Anglia, Norwich.

BibB, M. J., Freeman, R. F. \& Hopwood, D. A.
(1977). Physical and genetical characterisation of a second sex factor, SCP2, for Streptomyces coelicolor A3(2). Molecular and General Genetics 154, 155-166. 
Gerber, N. N. (1975). Prodigiosin-like pigments. CRC Critical Reviews in Microbiology 3, 469-485.

Gerber, N. N. \& Le Chevalier, M. P. (1976). Prodiginine (prodigiosin-like) pigments from Streptomyces and other aerobic actinomycetes. Canadian Journal of Microbiology 22, 658-667.

Hopwood, D. A. (1967). Genetic analysis and genome structure in Streptomyces coelicolor. Bacteriological Reviews 31, 373-403.

Hopwood, D. A., Chater, K. F., Dowding, J. E. \& Vivian, A. (1973). Advances in Streptomyces coelicolor genetics. Bactoriological Reviews 37, $371-405$.

KIRBY, R. \& Hopwood, D. A. (1977). Genetic determination of methylenomycin synthesis by the SCP1 plasmid of Streptomyces coelicolor A3(2). Journal of General Microbiology 98, 239-252.

MERRICK, M. J. (1976). A morphological and genetic mapping study of bald colony mutants of Stropto- myces coelicolor. Journal of General Microbiology 96, 299-315.

OKanishi, M., Suzuki, K. \& Umezawa, H. (1974). Formation and reversion of streptomycete protoplasts: cultural condition and morphological study. Journal of General Microbiology 80, 389-400.

RudD, B. A. M. \& Hopwood, D. A. (1979). Genetics of actinorhodin biosynthesis by Streptomyces coelicolor A3(2). Journal of General Microbiology 114, 35-43.

Wright, L. F. \& Hopwood, D. A. (1976a). Identification of the antibiotic determined by the SCP1 plasmid of Streptomyces coelicolor A3(2). Journal of General Microbiology 95, 96-106.

WRIGHT, L. F. \& Hopwood, D. A. (1976b). Actinorhodin is a chromosomally determined antibiotic in Streptomyces coelicolor A3(2). Journal of General Microbiology 96, 289-297. 